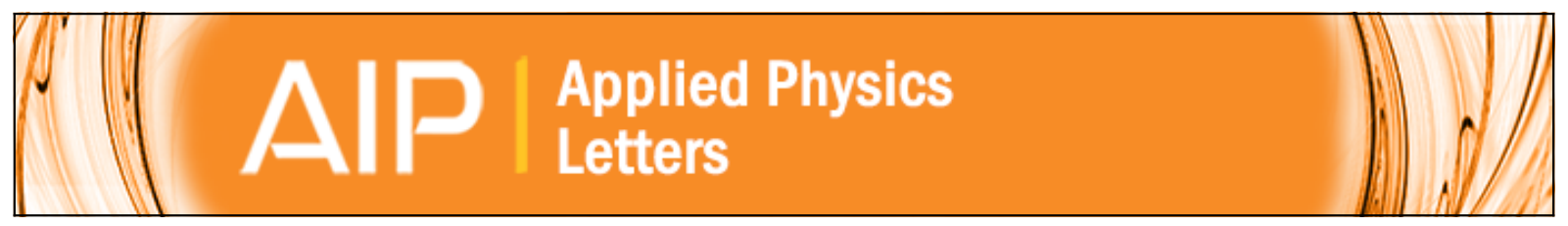

\title{
Ferromagnetic nanoclusters formed by Mn implantation in GaAs
}

O. D. D. Couto Jr., M. J. S. P. Brasil, F. likawa, C. Giles, C. Adriano, J. R. R. Bortoleto, M. A. A. Pudenzi, H. R. Gutierrez, and I. Danilov

Citation: Applied Physics Letters 86, 071906 (2005); doi: 10.1063/1.1863436

View online: http://dx.doi.org/10.1063/1.1863436

View Table of Contents: http://scitation.aip.org/content/aip/journal/apl/86/7?ver=pdfcov

Published by the AIP Publishing

\section{AlP}

\section{Create a profile. \\ Sign up today!}




\title{
Ferromagnetic nanoclusters formed by Mn implantation in GaAs
}

\author{
O. D. D. Couto, Jr., M. J. S. P. Brasil, and F. likawa \\ Instituto de Física "Gleb Wataghin," UNICAMP, C-6167, 13083-970, Campinas-SP, Brazil \\ C. Giles \\ Instituto de Física "Gleb Wataghin," UNICAMP, C-6167, 13083-970, Campinas-SP, Brazil and Laboratório \\ Nacional de Luz Síncrotron, CP-6192, 13084-971, Campinas-SP, Brazil \\ C. Adriano, J. R. R. Bortoleto, ${ }^{a}$ M. A. A. Pudenzi, and H. R. Gutierrez') \\ Instituto de Física “Gleb Wataghin," UNICAMP, C-6167, 13083-970, Campinas-SP, Brazil \\ I. Danilov ${ }^{\mathrm{c})}$ \\ Departamento de Física, UFRGS, CP-15051, 91501-970, Porto Alegre-RS, Brazil
}

(Received 24 September 2004; accepted 16 December 2004; published online 8 February 2005)

\begin{abstract}
Ferromagnetic clusters were incorporated into GaAs samples by Mn implantation and subsequent annealing. The composition and structural properties of the Mn-based nanoclusters formed at the surface and buried into the GaAs sample were analyzed by x-ray and microscopic techniques. Our measurements indicate the presence of buried MnAs nanoclusters with a structural phase transition around $40{ }^{\circ} \mathrm{C}$, in accord with the first-order magneto-structural phase transition of bulk MnAs. We discuss the structural behavior of these nanoclusters during their formation and phase transition, which is an important point for technological applications. (C) 2005 American Institute of Physics.
\end{abstract}

[DOI: $10.1063 / 1.1863436$ ]

The possibility of preparing submicron ferromagnets in semiconductors is of strong interest to the development of new spintronic devices. ${ }^{1,2}$ The formation of Mn-based nanoclusters in GaAs samples by ion implantation and subsequent annealing $^{3-5}$ is a relatively simple technique compared to the growth of (GaAs)Mn diluted material by epitaxial techniques, with the advantages of easy control of the Mn relative density and the possibility of spatial confinement with mask patterning. Both GaMn (Refs. 3,4) and MnAs (Refs. $6,7)$ present room temperature ferromagnetism. MnAs exhibits a simultaneous structural and magnetic first-order phase transition at $\sim 40{ }^{\circ} \mathrm{C}$, from hexagonal-ferromagnetic ( $\alpha$-phase) to orthorhombic-paramagnetic ( $\beta$-phase) ${ }^{6,7} \mathrm{~A}$ remarkable aspect from the structural point of view is that the $\mathrm{Mn}$ nanostructures obtained by ion implantation present good crystal quality despite the structural difference between those structures and the GaAs matrix. Both MnAs (orthorhombic/hexagonal) $^{6,7}$ and GaMn (icosahedral) ${ }^{8}$ exhibit lattice structures that are markedly different from the cubic GaAs. The dynamics of formation of Mn nanoclusters remains mostly unknown. A detailed analysis of this system requires several complex techniques.

We investigate the structure and composition of magnetic nanoclusters created by $\mathrm{Mn}$ implantation on GaAs samples with special emphasis on MnAs clusters and their properties. Our results indicate the presence of MnAs nanoclusters embedded in the GaAs sample presenting a structural phase transition typical of bulk MnAs with a substantial variation of their lattice constants despite severe threedimensional constraints imposed by the GaAs matrix.

\footnotetext{
a) Present address: UNESP, U. D. Sorocaba/Iperó, Sorocaba 18087-180, SP Brazil.

${ }^{b)}$ Present address: Penn State University, Department of Physics, Ins. Mat. Res., University Park, Pennsylvania 16802.

${ }^{c)}$ Present address: Nizhny Novgorod Sate University, 603950 Nizhny Novgorod, Russia.
}

Semi-insulating GaAs (001) substrates were implanted with $\mathrm{Mn}^{+}$ions with $200 \mathrm{keV}$ and doses of $1-3 \times 10^{16} \mathrm{~cm}^{-2}$. Subsequent rapid thermal annealing (RTA) was performed at $700-900{ }^{\circ} \mathrm{C}$ for $2-60 \mathrm{~s}$ in $\mathrm{Ar}$ atmosphere with the samples covered with a Si plate to minimize As evaporation. X-ray diffraction measurements were performed at the Brazilian National Synchrotron Light Laboratory (LNLS) using a 7600 $\mathrm{eV}$ energy beam with the sample temperature controlled by a Peltier thermoelectric device. Atomic force microscopy (AFM) images were used to spatially probe the topography of the samples surface. For structural and composition analysis, cross-sectional transmission electron microscopy (TEM) and energy dispersive $\mathrm{x}$-ray (EDX) measurements were employed with a JEM-3010 URP microscope operating at 300 $\mathrm{kV}$. The depth distribution of Mn was investigated by secondary ion mass spectrometry (SIMS) measurements.

After annealing we observed a strong diffusion of $\mathrm{Mn}$ towards the sample surface resulting in the formation of surface clusters with a ferromagnetic character. The AFM image from a typical sample [Fig. 1(a)] shows nanoclusters with a mean diameter of $\sim 200 \mathrm{~nm}$, mean height of $\sim 15 \mathrm{~nm}$ and average density of $\sim 2.5 \times 10^{8} \mathrm{~cm}^{-2}$. TEM measurements revealed that the surface nanoclusters may assume various distinct shapes, as shown by the cross-sectional images presented in Figs. 1(b) and 1(c). Point 1 in Fig. 1(b) marks a shallow surface structure $(\sim 20 \mathrm{~nm}$ height $)$, whereas the structure presented on Fig. 1(c) (point 4) is much larger ( $\sim 70 \mathrm{~nm}$ height) and shows more defined facets. Furthermore, we observe the presence of buried nanoclusters as a series of nearly circular structures with diameters of the order of $\sim 50 \mathrm{~nm}$ along a line parallel to the sample surface [point 3 in Fig. 1(b)]. The buried nanoclusters are formed at a depth of $\sim 150 \mathrm{~nm}$ below the sample surface. This agrees with the mean projected range of the Mn ions simulated by the TRIM code and with the local maximum of Mn concentration observed by SIMS even for annealed samples. Similar results were previously reported. ${ }^{9}$ 


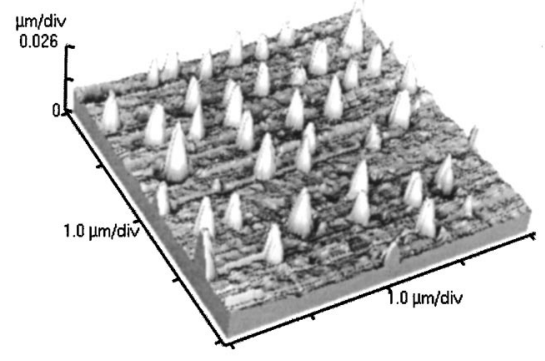

(a)

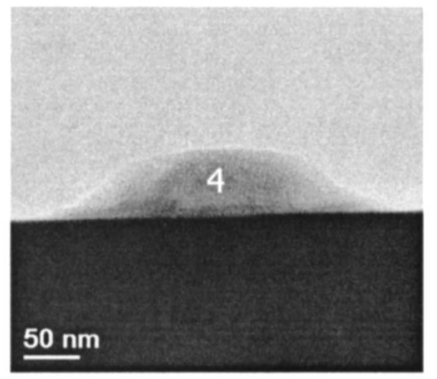

(c)

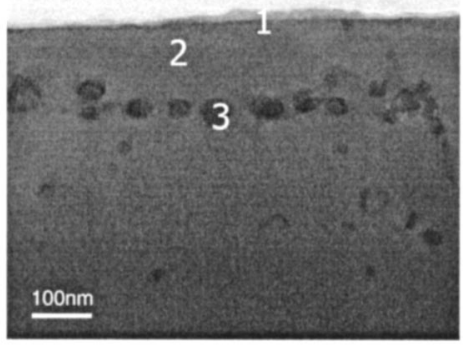

(b)

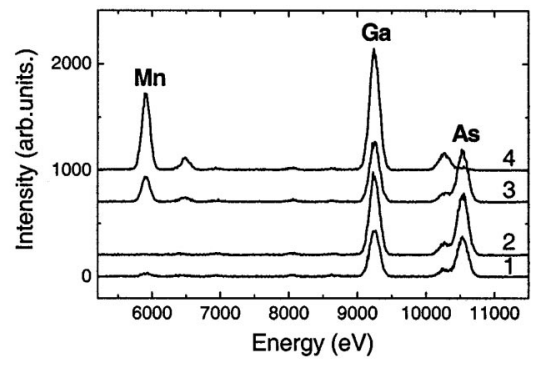

(d)

FIG. 1. (a) AFM image showing the nanoclusters formed at the surface of a $\mathrm{Mn}$ implanted GaAs sample with subsequent annealing. (b) and (c) Crosssectional TEM image on Mnimplanted samples. The points indicated by numbers correspond to the positions analyzed by EDX. (d) EDX emission spectra for the points marked at the TEM images.

EDX spectra at the points marked by numbers on Figs. 1(b) and 1(c) are presented in Fig. 1(d). The composition analysis is semiqualitative due to the large uncertainty originating from the signal integration over a relatively large volume, defined by the area of the electron beam probe (diameter of $\sim 20 \mathrm{~nm}$ ) and the sample thickness prepared for EDX measurements $(\sim 100 \mathrm{~nm})$. When this volume is larger than that of the nanocluster, the signal includes a non-negligible contribution from the surrounding GaAs matrix that must be taken into account. Emission from Mn atoms was observed for all nanoclusters, but no Mn emission was detected from regions adjacent to nanostructures [such as point 2 in Fig. 1(b)] indicating that the $\mathrm{Mn}$ is strongly concentrated at the nanoclusters. We estimated the Ga/As ratio of the nanoclusters considering the ratio between the emission intensities from $\mathrm{Ga}$ and As for their surrounding regions as a reference of a pure GaAs material and taking into account their dimensions relative to the probe volume. The buried structures [point 3 in Fig. 1(a)] present a relatively strong EDX signal from $\mathrm{Mn}$ atoms and a $\mathrm{Ga} / \mathrm{As}$ composition ratio smaller than that of the GaAs reference. The $\mathrm{Ga}$ emission corresponds approximately to the signal expected from the surrounding GaAs region of those relatively small clusters. Therefore they must be composed of pure MnAs or a (Ga,As)Mn alloy with a residually low Ga concentration. On the other hand, the EDX spectrum for the larger surface structure [point 4 in Fig. 1(c)] shows almost no emission from As atoms, indicating that those structures are composed mainly of GaMn. The uncertainty is obviously larger for the smaller surface structures [point 1 at Fig. 1(b)] for which EDX results indicate a (Ga,As)Mn alloy with a As concentration slightly larger than $\mathrm{Ga}$.

The inset of Fig. 2 shows a x-ray diffraction spectrum from a Mn implanted GaAs sample measured at room temperature in a grazing-incidence diffraction (GID) configuration. The dominant peak at $2 \theta=48.2^{\circ}$ corresponds to the GaAs (220) diffraction and the various additional peaks are attributed to $(\mathrm{Ga}, \mathrm{As}) \mathrm{Mn}$ alloys with different compositions. The peak at $2 \theta \sim 52.2^{\circ}$ coincides with the (1120) reflection of $\alpha$-MnAs, ${ }^{6,7}$ giving evidence to the presence of pure MnAs nanoclusters. Based on EDX results, we believe that those MnAs nanoclusters are buried in the GaAs matrix. Figure 2 shows $\mathrm{x}$-ray diffraction patterns for a heating cycle from 14 to $81{ }^{\circ} \mathrm{C}$ at this diffraction peak. At low temperatures the peak is at $\sim 52.15^{\circ}$; between $40^{\circ} \mathrm{C}$ and $50{ }^{\circ} \mathrm{C}$ it shifts to larger angles and for higher temperatures it is at $\sim 52.33^{\circ}$. All other diffraction peaks of the sample remain basically constant with temperature, showing only a slight shift to smaller angles, i.e., larger lattice parameters, due to thermal expansion. The behavior is reversed in a cooling cycle and is fully reproducible for various cycles.

The temperature dependence of the basal-lattice parameter of the hexagonal structure $\boldsymbol{a}$ obtained from the (11 $\overline{2} 0)$ MnAs diffraction peak is compared in Fig. 3 with the corresponding bulk MnAs values extracted from Ref. 6. The basal-lattice constant from the nanoclusters also presents a significant variation around the bulk phase transition tem-

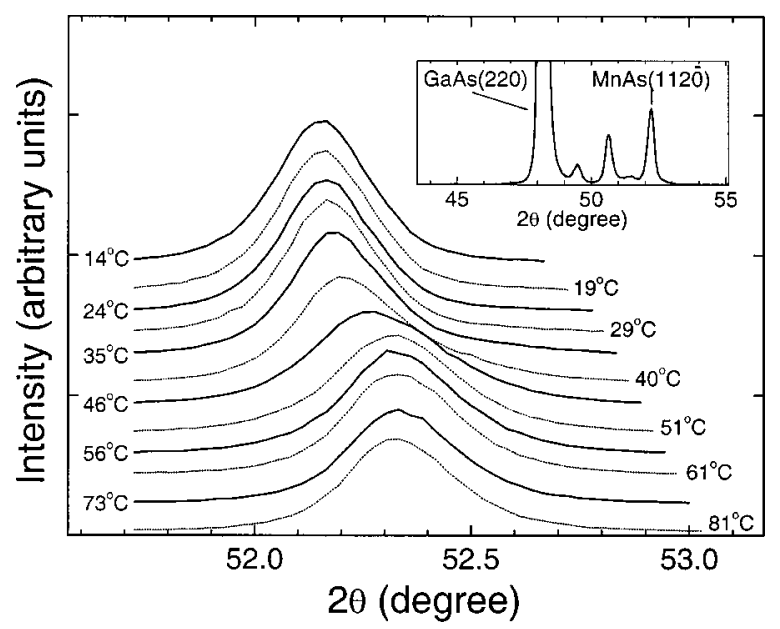

FIG. 2. Thermal variation of the x-ray diffraction patterns of the MnAs $(11 \overline{2} 0)$ plane from the nanoclusters. The inset shows a x-ray diffraction pattern obtained from the same sample at room temperature $\left(\sim 20^{\circ} \mathrm{C}\right)$ for a larger $2 \theta$ range. 


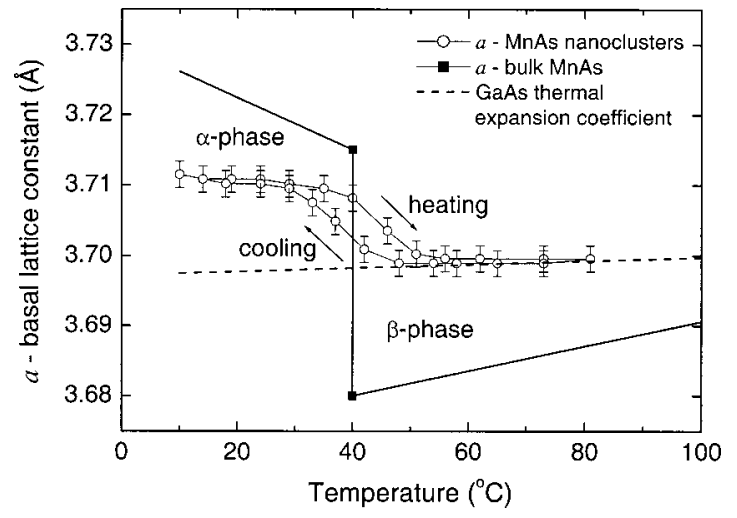

FIG. 3. Temperature dependence of the basal lattice constant $\boldsymbol{a}$ obtained for the MnAs nanoclusters (open circles) and for bulk MnAs (dashed line) (Ref. 6 ). The continuous line is a linear fit for the $\beta$-phase lattice parameter from the nanoclusters using an angular coefficient corresponding to the GaAs thermal expansion coefficient $\left(\sim 7 \times 10^{-6} / \mathrm{K}\right)$ (Ref. 13).

perature. The magnitude of the lattice parameter variation is, however, very different for the two systems. As compared to the bulk values, the value of $\boldsymbol{a}$ obtained for the nanoclusters is larger at the $\beta$-phase $\left(T>50{ }^{\circ} \mathrm{C}\right)$ and smaller at the $\alpha$-phase $\left(T<30^{\circ} \mathrm{C}\right)$, so that its variation during the phase transition $(\sim 0.3 \%)$ is much smaller than the corresponding bulk variation $(\sim 1 \%)$. Furthermore, the nanoclusters lattice parameter presents a thermal hysteresis, i.e., the phase transition occurs at higher temperatures during heating as compared to a cooling cycle, and is not very abrupt, which is attributed to the coexistence of the two phases. This coexistence can arise from a single nanostructure and/or a distribution of pure $\alpha$ - and $\beta$-phase nanostructures and its origin is still a subject of investigation. Both the coexistence and the hysteresis have been observed in previous works ${ }^{10-12}$ on thin MnAs films grown on GaAs and were attributed to strain effects.

We also point out that the thermal expansion/contraction of the MnAs nanoclusters when they are completely at $\alpha$-phase $\left(T<30^{\circ} \mathrm{C}\right)$ or $\beta$-phase $\left(T>50{ }^{\circ} \mathrm{C}\right)$ does not follow the corresponding bulk behavior, which is clearly observed in Fig. 3. Instead, the basal lattice parameter of the Mn nanoclusters shows a thermal variation similar to GaAs. The dashed line in Fig. 3 was plotted using the GaAs thermal expansion coefficient and fits quite well the thermal variation of the basal-lattice constant for $\beta$-phase nanoclusters. In order to understand this result, we must analyze the nanocluster formation. MnAs nanoclusters are formed during the thermal annealing at high temperatures $\left(700-900{ }^{\circ} \mathrm{C}\right)$, where all strain due to the distinct lattice structures between MnAs and GaAs must be released. As the sample is cooled, a strain should develop due to the large difference between the thermal expansion coefficients of MnAs $\left(\sim 6 \times 10^{-5} / \mathrm{K}\right)$ (Ref. 6) and GaAs $\left(\sim 7 \times 10^{-6} / \mathrm{K}\right) .{ }^{13}$ At relatively high temperatures, the strain can be efficiently accommodated due to the thermal energy of the system, but we expect that at a certain temperature the thermal energy becomes insufficient to re- lieve the strain. Below this temperature, the MnAs nanocluster must follow the GaAs thermal expansion coefficient and strain will therefore accumulate at the nanocluster. Since the GaAs thermal expansion coefficient is smaller than that of $\mathrm{MnAs}$, the basal-lattice constant from the MnAs nanostructures cooled to $\sim 80^{\circ} \mathrm{C}$ ( $\beta$-phase) should end up larger than that of bulk MnAs, as observed. The temperature from which strain starts to build up at the MnAs nanostructure can be estimated by the crossing point of the extrapolation of the bulk and the nanoclusters data, which gives $\sim 160{ }^{\circ} \mathrm{C}$, a very feasible value.

The fact that the first-order magneto-structural phase transition of MnAs persists for MnAs nanoclusters with dimensions of the order of $50 \mathrm{~nm}$ embedded in a GaAs matrix is a novel result that may have very interesting consequences. The expansion/contraction of buried MnAs nanoclusters during phase transition should be strongly constrained by the GaAs matrix. In fact, an ideal nanocluster surrounded by an infinitely larger GaAs matrix should not present a structural transition at all, being obliged to follow the thermal variation of the GaAs matrix. The weakened, but nonzero, basal-lattice constant variation displayed by the MnAs nanoclusters may be attributed to a residual degree of freedom from lattice defects formed around the nanocluster and a partial relief of strain to the surrounding GaAs material. Strain effects significantly affect the stability of the ferromagnetic $\alpha$-phase MnAs nanoclusters and they must therefore be considered in the development of device applications based on such nanostructures.

We kindly acknowledge the financial support from FAPESP, CAPES, and CNPq. TEM measurements were made at the LME laboratory of the Brazilian National Synchrotron Light Laboratory (LNLS), Campinas-SP, Brazil.

${ }^{1}$ G. A. Prinz, Science 250, 1092 (1990).

${ }^{2}$ G. A. Prinz, Phys. Today 48, 58 (1995).

${ }^{3}$ J. Shi, J. M. Kikkawa, R. Proksch, T. Schaeffer, D. D. Awschalom, G. Medeiros-Ribeiro, and P. M. Petroff, Nature (London) 377, 707 (1995).

${ }^{4}$ J. Shi, J. M. Kikkawa, D. D. Awschalom, G. Medeiros-Ribeiro, P. M. Petroff, and K. Babcock, J. Appl. Phys. 79, 5296 (1996).

${ }^{5}$ C. Chen, M. Cai, X. Wang, S. Xu, M. Zhang, X. Ding, and Y. Sun, J. Appl. Phys. 87, 9 (2000); 87, 5636 (2000).

${ }^{6}$ B. T. M. Willis and H. P. Rooksby, Proc. Phys. Soc. London, Sect. B 67, 290 (1954).

${ }^{7}$ R. H. Wilson and J. S. Kasper, Acta Crystallogr. 17, 95 (1964).

${ }^{8}$ J. P. Zhang, A. K. Cheetham, K. Sun, J. S. Wu, K. H. Kuo, J. Shi, and D. D. Awschalom, Appl. Phys. Lett. 71, 143 (1997).

${ }^{9}$ A. Serres, G. Benassayag, M. Respaud, C. Armand, J.-C. Pesant, A. Mari, Z. Liliental-Weber, and A. Claverie, Mater. Sci. Eng., B 101, 119 (2003); A. Serres, M. Respaud, G. Benassayag, C. Armand, J.-C. Pesant, A. Mari, Z. Liliental-Weber, and A. Claverie, Physica E (Amsterdam) 17, 371 (2003).

${ }^{10}$ V. M. Kaganer, B. Jenichen, F. Schippan, W. Braun, L. Däweritz, and K. H. Ploog, Phys. Rev. Lett. 85, 341 (2000).

${ }^{11}$ V. M. Kaganer, B. Jenichen, F. Schippan, W. Braun, L. Däweritz, and K. H. Ploog, Phys. Rev. B 66, 045305 (2002).

${ }^{12}$ F. Iikawa, P. V. Santos, M. Kästner, F. Schippan, and L. Däweritz, Phys. Rev. B 65, 205328 (2002).

${ }^{13}$ Landoldt-Börnstein, New Series, III/17a, 234. 